\title{
OPTIMAL CONTROL OF PWA SYSTEMS BY EXPLOITING PROBLEM STRUCTURE
}

\author{
M. Barić, P. Grieder, M. Baotić, M. Morari
}

\author{
Automatic Control Laboratory \\ Swiss Federal Institute of Technology \\ ETH Zentrum, ETLI 22, CH-8092, Zürich, Switzerland \\ baric / grieder / baotic / morari @control.ee.ethz.ch
}

\begin{abstract}
We present a novel algorithm for the computation of explicit optimal control laws for piecewise affine (PWA) systems with linear performance indices. The algorithm is based on dynamic programming (DP) and represents an extension of ideas initially proposed in (Kerrigan and Mayne, 2002; Baotić et al., 2003). Specifically, we show how to exploit the underlying geometric structure of the optimization problem in order to significantly improve the efficiency of the off-line computations. An extensive case study is provided, which clearly indicates that the algorithm proposed in this paper is preferable to other schemes published in the literature. Copyright (C)2005 IFAC.
\end{abstract}

Keywords: optimal control, dynamic programming, piecewise linear controllers,

\section{INTRODUCTION}

In the last decades, hybrid systems have been the subject of intensive research activities in the control community. In this paper we focus on a specific class of discrete time hybrid systems, namely, the class of constrained piecewise affine $(P W A)$ systems. PWA systems are defined by a series of affine systems whereby each affine dynamic is defined over a polyhedral set in the state-input space. PWA systems are important because they are equivalent to a broad class of hybrid systems (Heemels et al., 2001) and useful for modelling nonlinear systems (Sontag, 1981).

Constrained finite time optimal control (CFTOC) of PWA systems has been widely addressed in the literature, e.g. (Borrelli, 2003; Kerrigan and Mayne, 2002; Baotić et al., 2003; Mayne and Raković, 2002). The explicit off-line solution to the CFTOC problem can be obtained by solving a multi-parametric mixed-integer linear program (mpMILP) for linear performance indices (Dua and Pistikopoulos, 2000), or, in theory, a multiparametric mixed-integer quadratic program (mp-
MIQP) for a quadratic cost . In (Borrelli, 2003), the author proposed a computational scheme for obtaining quadratic optimal controllers for PWA systems which was based on dynamic programming (DP). For PWA systems and linear performance indices, a DP-based approach was introduced in (Kerrigan and Mayne, 2002), where the authors consider the optimal control of PWA systems affected by bounded disturbances. The scheme proposed in (Kerrigan and Mayne, 2002) uses a series of multi-parametric linear programs (mpLPs) instead of one mpMILP. Though giving general ideas about the DP-based concept and the character of the solution for linear performance objectives, computational issues are not addressed in (Kerrigan and Mayne, 2002). An implementation of the DP-based algorithm in (Kerrigan and Mayne, 2002) for performance indices based on 1 and $\infty$ norms is described in detail in (Baotić et al., 2003). Practical experience with these computation schemes shows that for PWA systems of higher dimensions and a large number of affine dynamics, the off-line computation of the explicit 
optimal control law may become too demanding to be applicable in practice.

Therefore, in this paper, we address the efficiency of the off-line computation of the CFTOC law. We show how to exploit problem convexity, such that fewer mpLPs need to be solved. For this new formulation, we show how to reduce the number of constraints defining polyhedral critical regions in the explicit control law by exploiting region adjacency information. Finally, we present an extensive case study in which we compare the runtime of the algorithm in (Kerrigan and Mayne, 2002; Baotić et al., 2003) with the runtime of the algorithm proposed here.

\section{PROBLEM STATEMENT AND PRELIMINARY RESULTS}

In this section we will define the CFTOC problem and give preliminary results which characterize its explicit solution. Also, we will shortly describe the DP-based algorithm in (Baotić et al., 2003).

\subsection{Problem Statement and Properties of the Solution}

Constrained discrete-time PWA systems are defined by the following set of equations:

$$
\begin{gathered}
\mathbf{x}(k+1)=f_{\mathrm{PWA}}(\mathbf{x}(k), \mathbf{u}(k)) \\
=\mathbf{A}^{\{i\}} \mathbf{x}(k)+\mathbf{B}^{\{i\}} \mathbf{u}(k)+\mathbf{f}^{\{i\}}, \\
\quad \text { if }\left[\begin{array}{l}
\mathbf{x}(k) \\
\mathbf{u}(k)
\end{array}\right] \in \mathcal{D}^{\{i\}}, \\
\mathcal{D}^{\{i\}}:=\left\{\left[\begin{array}{l}
\mathbf{x} \\
\mathbf{u}
\end{array}\right] \mid\left[\left(\mathbf{P}^{x}\right)^{\{i\}}\left(\mathbf{P}^{u}\right)^{\{i\}}\right]\left[\begin{array}{l}
\mathbf{x} \\
\mathbf{u}
\end{array}\right] \leq\left(\mathbf{P}^{0}\right)^{\{i\}}\right\}
\end{gathered}
$$

where $k \geq 0, \mathbf{x} \in \mathbb{R}^{n}$ is the state vector, $\mathbf{u} \in \mathbb{R}^{m}$ is the control vector and $\left\{\mathcal{D}^{\{i\}}\right\}_{i=1}^{D}$ is a bounded polyhedral partition of $(\mathbf{x}, \mathbf{u}) \subset \mathbb{R}^{n+m}$ space. The constraints $\left(\mathbf{P}^{x}\right)^{\{i\}} \mathbf{X}+\left(\mathbf{P}^{u}\right)^{\{i\}} \mathbf{u} \leq\left(\mathbf{P}^{0}\right)^{\{i\}}$ define both regions in which a particular state update equation is valid as well as constraints on the state and input variables. Consider the following cost function:

$$
\begin{aligned}
& J\left(\mathbf{U}_{0}^{N-1}, \mathbf{x}(0)\right):=\left\|\mathbf{P}_{N} \mathbf{x}(N)\right\|_{\ell} \\
&+\sum_{k=0}^{N-1}\|\mathbf{Q} \mathbf{x}(k)\|_{\ell}+\|\mathbf{R u}(k)\|_{\ell},
\end{aligned}
$$

where $N$ is the time horizon, $\mathbf{P}_{N}$ is a matrix defining the weight on the terminal state $\mathbf{x}(N)$, $\|\cdot\|_{\ell}$ denotes the vector norm with $\ell \in\{1, \infty\}$ and $\mathbf{U}_{0}^{N-1}=\left[\mathbf{u}^{T}(0), \ldots, \mathbf{u}^{T}(N-1)\right]^{T} \in \mathbb{R}^{m N}$ is the vector of control inputs. The goal of CFTOC is to minimize the cost function (2), i.e.:

$$
\begin{aligned}
& J_{N}^{*}(\mathbf{x}(0)):=\min _{\mathbf{U}_{0}^{N-1}} J_{N}\left(\mathbf{U}_{0}^{N-1}, \mathbf{x}(0)\right), \\
& \text { subj. to }\left\{\begin{array}{l}
\mathbf{x}(k+1)=f_{\text {PWA }}(\mathbf{x}(k), \mathbf{u}(k)), \\
\mathbf{x}(N) \in \mathcal{T}_{\text {set }},
\end{array}\right.
\end{aligned}
$$

where $\mathcal{T}_{\text {set }}$ is a terminal set, i.e. the set of admissible states at the final time instance $N$. The following theorem characterizes the solution of the CFTOC problem (1)-(3).

Theorem 2.1. ((Borrelli, 2003), Theorem 7.4.1). The solution to the optimal control problem (1)-(3) with $\ell \in\{1, \infty\}$ is a polyhedral piecewise affine $(P P W A)$ (affine in every polyhedron) state feedback control law of the form:

$$
\mathbf{u}^{*}(k)=\mathbf{F}_{k}^{\{i\}} \mathbf{x}(k)+\mathbf{G}_{k}^{\{i\}} \quad \text { if } \mathbf{x}(k) \in \mathcal{R}_{k}^{\{i\}},
$$

where $\mathcal{R}_{k}^{\{i\}}, i=1, \ldots, R_{k}$ are polyhedra defining a polyhedral partition of the set $\mathcal{X}_{k}$ of feasible states $\mathbf{x}(k)$ at time step $k=0, \ldots, N-1$.

The PPWA solution to the CFTOC problem can be obtained by formulating the problem as a DP and solving a number of mpLPs (Kerrigan and Mayne, 2002; Baotić et al., 2003). In each mpLP, the state vector $\mathbf{x}$ is considered to be a vector of parameters and the control input $\mathbf{u}$ is the optimization variable. For further discussion, we will need the following result related to the character of the solution of an mpLP:

Theorem 2.2. ((Borrelli, 2003), Theorems 1.3.3-4). Consider the mpLP:

$$
\begin{aligned}
J^{*}(\mathbf{x})= & \min _{\mathbf{z}} J(\mathbf{z}, \mathbf{x})=\mathbf{c}^{T} \mathbf{z}, \\
& \text { subj. to } \mathbf{G z} \leq \mathbf{S x}+\mathbf{W},
\end{aligned}
$$

where $\mathbf{z} \in \mathbb{R}^{s}$ is a vector of optimization variables, $\mathbf{x} \in \mathbb{R}^{n}$ is a vector of parameters, $J(\mathbf{z}, \mathbf{x}): \mathbb{R}^{s} \times$ $\mathbb{R}^{n} \rightarrow \mathbb{R}$ is the objective function and $\mathbf{G} \in \mathbb{R}^{q \times s}$, $\mathbf{S} \in \mathbb{R}^{q \times n}, \mathbf{W} \in \mathbb{R}^{q}$. Let $\mathcal{P}^{*}$ be the set of parameters $\mathbf{x}$ for which the linear program (5) has a finite optimal solution. Then:

i) $\mathcal{P}^{*}$ is a closed polyhedral set in $\mathbb{R}^{n}$,

ii) The value function $J^{*}(\mathbf{x})$ is convex and PPWA over $\mathcal{P}^{*}$, i.e.

$$
J^{*}(\mathbf{x})=\boldsymbol{\alpha}^{\{i\}} \mathbf{x}+\beta^{\{i\}}, \quad \text { if } \quad \mathbf{x} \in \mathcal{C} \mathcal{R}^{\{i\}},
$$

where $\left\{\mathcal{C} \mathcal{R}^{\{i\}}\right\}_{i=1}^{R}$, are polyhedra and $\mathcal{P}^{*}=$ $\bigcup_{i=1}^{R} \mathcal{C} \mathcal{R}^{\{i\}}$.

Polyhedra $\mathcal{C} \mathcal{R}^{\{i\}}$, defining the partition of the feasible set of parameters $\mathcal{P}^{*}$, are known in the theory of parametric programming as critical regions. To make a clear distinction between the solution of a single mpLP and the general solution to a CFTOC problem (1)-(3), i.e. between critical regions $\mathcal{C} \mathcal{R}^{\{i\}}$, and regions $\mathcal{R}_{k}^{\{k\}}$ in Theorem 2.1, we will refer to $\mathcal{R}_{k}^{\{i\}}$ as controller regions. Note also that the convexity results given in Theorem 2.2 are valid only for the solution of a single mpLP. The set of feasible parameters and the value function of the CFTOC problem (1)-(3) are in general 
non-convex. For more details about the theory of mpLP, the reader is referred to (Gal, 1995; Borrelli, 2003).

\subsection{DP-Based Algorithm with Affine "Cost-To-Go"}

In this subsection we briefly describe the DPbased algorithm previously published in (Kerrigan and Mayne, 2002; Baotić et al., 2003). Problem (3) can be stated as an equivalent dynamic program:

$$
\begin{aligned}
J_{k}^{*}(\mathbf{x}(k)):= & \min _{\mathbf{u}(k)}\|\mathbf{Q} \mathbf{x}(k)\|_{\ell}+\|\mathbf{R u}(k)\|_{\ell} \\
& +J_{k+1}^{*}\left(f_{\mathrm{PWA}}(\mathbf{x}(k), \mathbf{u}(k))\right),
\end{aligned}
$$

subj. to $f_{\mathrm{PWA}}(\mathbf{x}(k), \mathbf{u}(k)) \in \mathcal{X}_{k+1}$,

for $k=N-1, \ldots, 0$, with boundary conditions

$$
\mathcal{X}_{N}=\mathcal{T}_{\text {set }}, \text { and } J_{N}^{*}(\mathbf{x})=\left\|\mathbf{P}_{N} \mathbf{x}\right\|_{\ell},
$$

where

$$
\mathcal{X}_{k}=\left\{\mathbf{x} \in \mathbb{R}^{n} \mid \exists \mathbf{u}, f_{\mathrm{PWA}}(\mathbf{x}, \mathbf{u}) \in \mathcal{X}_{k+1}\right\}
$$

is the set of all initial states for which the problem $(7)$ is feasible. The term $J_{k+1}^{*}(\mathbf{x}(k+1))$, representing the cost of all future control actions, is commonly referred to as "Cost-To-Go". Since the set $\mathcal{X}_{k+1}$ and $J_{k+1}^{*}(\mathbf{x}(k+1))$ are in general nonconvex for PWA systems, mpLP solvers cannot be applied directly to solve (7). Instead, the nonconvex problem (7) is split into a number of convex sub-problems by formulating one mpLP for each controller region obtained at the previous iteration of the DP:

$$
\begin{aligned}
& \qquad \begin{aligned}
J_{k}^{*}(\mathbf{x}(k)):= & \min _{\mathbf{u}(k)}\|\mathbf{Q} \mathbf{x}(k)\|_{\ell}+\|\mathbf{R u}(k)\|_{\ell}+ \\
& +\boldsymbol{\alpha}_{k+1}^{\{r\}} \mathbf{x}(k+1)+\beta_{k+1}^{\{r\}},
\end{aligned} \\
& \text { s. t. } \mathbf{x}(k+1)=f_{\mathrm{PWA}}(\mathbf{x}(k), \mathbf{u}(k)) \in \mathcal{R}_{k+1}^{\{r\}},
\end{aligned}
$$

In (9) the non-convex PWA "Cost-To-Go" has been replaced by the affine term $J_{k+1}^{*}=\boldsymbol{\alpha}_{k+1}^{\{r\}} \mathbf{x}(k+$ $1)+\beta_{k+1}^{\{r\}}$. This way, problem (9) needs to be solved for all controller regions $\left\{\mathcal{R}_{k+1}^{\{r\}}\right\}_{r=1}^{R_{k+1}}$ and all dynamics $\left\{\mathcal{D}^{\{i\}}\right\}_{i=1}^{D}$. The result of each of $R_{k+1} \cdot D$ mpLPs (9) is a closed polyhedral partition $\mathcal{P}_{k}^{\{i\}}$, the union of which is the set of feasible parameters in step $k$, i.e. $\mathcal{X}_{k}=\bigcup_{i=1}^{R_{k+1} \cdot D} \mathcal{P}_{k}^{\{i\}}$. Note that the partitions $\mathcal{P}_{k}^{\{i\}}$ will overlap, in general. In order to obtain a suitable target partition for the next step of the DP, it is therefore necessary to compare the $\operatorname{cost} J_{k}^{*}(\mathbf{x})$ wherever controller regions overlap and to remove the controller regions which are not cost optimal. Detection of overlapping critical regions within partitions $\mathcal{P}_{k}^{\{i\}}$ and comparison of the cost are done by solving a (possibly large) number of LPs ( (Borrelli, 2003), pg. 158-160). The computational complexity of removing overlaps grows exponentially with the number of regions covering any given state $\mathbf{x}$. As the number of mpLPs solved in one step of the DP grows with each iteration of the DP, the number of overlaps grows as well. Thus, a significant amount of time is spent on the removal of overlaps.

\section{DYNAMIC PROGRAMMING WITH CONVEX PWA "COST-TO-GO"}

In this section we present the main contribution of the paper. We show how to reformulate the DP problem presented in the previous section such that number of mpLPs which need to be solved is reduced. Before stating the algorithm formally, we will give a simple example to illustrate the key ideas.

Consider a one-dimensional PWA system and a single DP iteration of the CFTOC problem for the $i$-th dynamic of the system:

$$
J_{k}^{*}(x(k)):=\min _{u(k)} J_{k+1}^{*}(x(k+1)),
$$

$$
\text { s.t. } x(k+1)=f_{\mathrm{PWA}}(x(k), u(k)) \in \mathcal{X}_{k+1}
$$

For the sake of simplicity, we consider only the minimization of the "cost-to-go" $J_{k+1}^{*}(x(k+1))$. The target set $\mathcal{X}_{k+1}$ is assumed to be convex and consisting of two subsets (see Fig. 1):

$$
\begin{aligned}
& \mathcal{X}_{k+1}^{\{1\}}:=\left\{x \mid x \in \mathcal{X}_{k+1} \wedge x \leq x_{c}\right\}, \\
& \mathcal{X}_{k+1}^{\{2\}}:=\left\{x \mid x \in \mathcal{X}_{k+1} \wedge x \geq x_{c}\right\}
\end{aligned}
$$

We further assume that $J_{k+1}^{*}$ is non-convex on $\mathcal{X}_{k+1}$ and affine in each of the subsets. The constraints (10b) define a polyhedron $\Pi$ in $(x, u)$ space (Fig. 1), the projection of which to the $x$ space defines the set of parameters (system states) $\mathcal{P}$ for which the optimization problem (10) is feasible. We split this non-convex problem into two subproblems by considering each segment of the target set separately, as explained in Subsection 2.2. A cut $x(k+1)=x_{c}$ in $(x, u)$-space

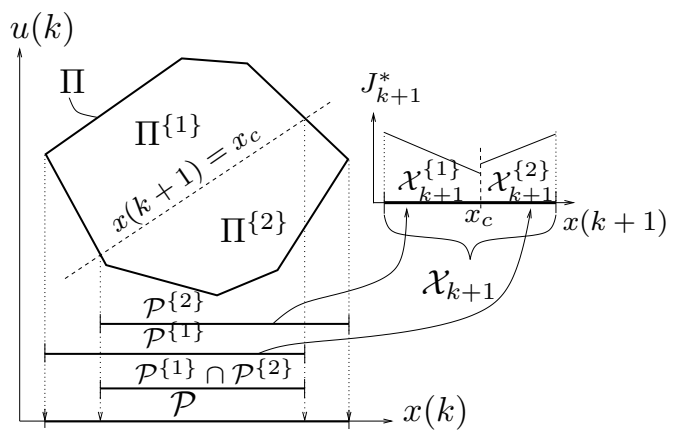

Fig. 1. DP-based algorithm with affine "cost-togo": for two adjacent target sets, two overlapping partitions $\mathcal{P}\{1\}$ and $\mathcal{P}^{\{2\}}$ are generated.

separates polyhedron $\Pi$ into two polyhedra $\Pi^{\{1\}}$ and $\Pi^{\{2\}}$ (see Fig. 1). Projections of these polyhedra to $x$-space define sets $\mathcal{P}^{\{1\}}$ and $\mathcal{P}^{\{2\}}$, which represent sets of feasible states for each mpLP 
subproblem. In general, the cut introduced by the additional constraint $x(k+1)=x_{c}$ separates the polyhedron $\Pi$ in such a way that the sets $\mathcal{P}^{\{1\}}$ and $\mathcal{P}^{\{2\}}$ overlap. On the other hand, if $J_{k+1}^{*}$ is convex PWA in $\mathcal{X}_{k+1}$, one can formulate the problem as a single $m p L P$ and obtain the solution as a set of non-overlapping critical regions whose union is $\mathcal{P}$. This will be shown in the following section.

\subsection{Dynamic Programming with Convex PWA "Cost-To-Go"}

Consider the DP formulation of the CFTOC problem (7) and assume that a terminal set is given by:

$$
\mathcal{X}_{N}=\mathcal{T}_{\text {set }}=\bigcup_{c=1}^{C_{N}} \mathcal{P}_{N}^{\{c\}},
$$

where $\mathcal{P}_{N}^{\{c\}}$ are closed polyhedral sets. Furthermore, assume that the value functions $\left(J_{N}^{*}\right)^{\{c\}}$ defined over each subset $\mathcal{P}_{N}^{\{c\}}$ are convex PPWA, i.e.:

$$
\left(J_{N}^{*}\right)^{\{c\}}(\mathbf{x})=\boldsymbol{\alpha}^{\{r, c\}} \mathbf{x}+\beta^{\{r, c\}}, \text { if } \mathbf{x} \in \mathcal{R}_{N}^{\{r, c\}}
$$

where $r=1, \ldots, R_{N}^{\{c\}}$ is the number of polyhedral regions in $\mathcal{P}_{N}^{\{c\}}=\bigcup_{r=1}^{R_{N}^{\{c\}}} \mathcal{R}_{N}^{\{r, c\}}$.

Being convex and PWA affine, each $\left(J_{N}^{*}\right)^{\{c\}}$ can be written as (Schechter, 1987):

$$
\left(J_{N}^{*}\right)^{\{c\}}(\mathbf{x})=\max _{r \in\left\{1, \ldots, R_{N}^{\{c\}}\right\}} \boldsymbol{\alpha}^{\{r, c\}} \mathbf{x}+\beta^{\{r, c\}},
$$

and the problem:

$$
\min _{\mathbf{x}}\left(J_{N}^{*}\right)^{\{c\}}(\mathbf{x}), \quad \text { s.t. } \mathbf{x} \in \mathcal{P}_{N}^{\{c\}}
$$

can be equivalently formulated as:

$$
\begin{array}{ll} 
& \min _{\gamma} \gamma \\
\text { s.t. } & \boldsymbol{\alpha}^{\{r, c\}} \mathbf{X}+\beta^{\{r, c\}} \leq \gamma,
\end{array}
$$

where $r=1, \ldots, R_{N}^{\{c\}}$. This is a well-known minimax principle for the minimization of PWA convex functions.

By taking into account Theorem 2.2, the nonconvex problem (7) can be split into a number of $c=1, \ldots, C_{k+1}$ mpLP subproblems of the following form:

$$
\begin{gathered}
\left(J_{N}^{*}\right)^{\{c\}}(\mathbf{x}):=\min _{\mathbf{u}, \gamma}\|\mathbf{Q} \mathbf{x}\|_{\ell}+\|\mathbf{R u}\|_{\ell}+\gamma, \\
\text { s.t. } \mathbf{x}(k+1)=f_{P W A}(\mathbf{x}(k), \mathbf{u}(k)) \in \mathcal{P}_{k+1}^{\{c\}}, \\
\boldsymbol{\alpha}^{\{r, c\}} \mathbf{x}(k+1)+\beta^{\{r, c\}} \leq \gamma, \\
\forall r \in\left\{1, \ldots, R_{k+1}^{\{c\}}\right\},
\end{gathered}
$$

where $C_{k+1}$ denotes the number of polyhedral target partitions $\mathcal{P}_{k+1}^{\{c\}}$ from iteration $k+1$ and $R_{k+1}^{\{c\}}$ corresponds to the number of polyhedral regions in $\mathcal{P}_{k+1}^{\{c\}}$. Hence, it is necessary to solve $C_{k}=C_{k+1} \cdot D$ mpLPs in order to obtain the solution to (15). The $C_{k}$ resulting partitions will overlap, in general, and a comparison of the mpLP solutions needs to be performed in order to get the solution of the original CFTOC problem, i.e. it is still necessary to remove overlaps in the partitions. So far, the only difference to the scheme described in Section 2.2 is that we consider only large convex controller partitions $\mathcal{P}_{k}^{\{c\}}$ as target sets, instead of the smaller convex controller regions. In order to avoid exponential growth of the number of partitions $\mathcal{P}_{k}^{\{c\}}$ with each iteration (mpLPs which need to be solved in each step of the DP), it is advisable to remove partitions which do not contain any optimal critical region, i.e. if for all $\mathbf{x} \in \mathcal{P}_{k}^{\{n\}}$ there exists a partition $\mathcal{P}_{k}^{\{m\}}$ and a corresponding value function $\left(J_{k}^{*}\right)^{\{m\}}$ such that $\mathbf{x} \in \mathcal{P}_{k}^{\{m\}}$ and $\left(J_{k}^{*}(\mathbf{x})\right)^{\{n\}}>\left(J_{k}^{*}(\mathbf{x})\right)^{\{m\}}$, then the partition $\mathcal{P}_{k}^{\{n\}}$ can be removed. Detection of suboptimal partitions can be done by using the value functions $\left(J_{k}^{*}\right)^{\{c\}}$ as a selection criterion and solving a (possibly large) number of LPs. Note that in the worst-case, it may not be possible to discard any partitions.

Comparing the new algorithm (15) to the approach in Section 2.2, it is obvious that the mpLPs in (15) are more complex, since the number of constraints is (considerably) higher due to the introduction of value function constraints (15c). As a remedy, we propose the following scheme based on region adjacency information.

\subsection{Constraint Reduction Using Adjacency List}

Using algebraic manipulations, the mpLP (15) can be put into the form (5). For further discussion we will need the notion of active constraints.

Definition 1. (Active Constraints). The set of active constraints $\mathcal{A}(\mathbf{x})$ for a given state $\mathbf{x}$ of problem (5) is defined as:

$$
\begin{array}{r}
\mathcal{A}(\mathbf{x}):=\left\{i \in \mathcal{I} \mid \forall \mathbf{z}: J_{k}(\mathbf{x}, \mathbf{z})=J_{k}^{*}(\mathbf{x}),\right. \\
\left.\mathbf{G}_{(i)} \mathbf{z}-\mathbf{S}_{(i)} \mathbf{x}-\mathbf{W}_{(i)}=0\right\}
\end{array}
$$

where $\mathbf{G}_{(i)}, \mathbf{S}_{(i)}$ and $\mathbf{W}_{(i)}$ denote the $i$-th row of matrices $\mathbf{G}, \mathbf{S}$ and $\mathbf{W}$ respectively, and $\mathcal{I}=$ $\{1, \ldots, q\}$.

Critical regions, defining the solution of the mpLP, are constructed as follows. For a given state (parameter) $\mathbf{x}^{*}$ an LP is solved and a set of active constraints $\mathcal{A}\left(\mathrm{x}^{*}\right)$ is identified. For the construction of the critical region, the set of inactive constraints $\mathcal{N}\left(\mathbf{x}^{*}\right)=\mathcal{I} \backslash \mathcal{A}\left(\mathbf{x}^{*}\right)$ is used, defining $q_{\mathcal{N}}$ half-spaces whose intersection describes the polyhedral critical region, i.e. the cardinality of $\mathcal{N}$ is $q_{\mathcal{N}}$. In order to obtain the minimal representation of the critical region, all redundant half-spaces need to be removed, so that the final 
representation of the critical region is defined as an intersection of $q_{\mathcal{N}}^{*} \leq q_{\mathcal{N}}$ half-spaces. This procedure requires the solution to $q_{\mathcal{N}}$ LPs per critical region (Borrelli, 2003). In our case, $q_{\mathcal{N}}$ increases with the number of value function constraints, i.e. the number of critical regions in the target partitions from the previous step of the DP, which may grow exponentially with each DP iteration. In the following we show how to reduce the number of initial half-spaces $q_{\mathcal{N}}$ by using region adjacency information, and thus significantly decrease the number of LPs which need to be solved per mpLP subproblem.

Before proceeding further, it is necessary to point out that, in the strict sense, critical regions are open sets. However, for any practical computation and analysis, a critical region is usually replaced by its closure. In the rest of the text, when speaking of a critical region, we will consider its closure.

Definition 2. (Adjacent regions). Polyhedral critical regions $\mathcal{C} \mathcal{R}^{\{i\}}$ and $\mathcal{C} \mathcal{R}^{\{j\}}$ are called adjacent if they share a common facet.

In the following, the term adjacent constraints will be used for value function constraints (15c) which correspond to adjacent critical regions. Let $\mathcal{A}\left(\mathrm{x}^{*}\right)$ be a set of active constraints for a given $\mathbf{x}^{*} \in$ $\mathcal{C R}_{k}^{\{l\}}$, where $\mathcal{C} \mathcal{R}_{k}^{\{l\}}$ is the critical region whose polyhedral representation we want to compute. The critical region $\mathcal{C R}_{k}^{\{l\}}$ can be obtained as a projection from $(\mathbf{x}, \mathbf{z})$-space to $\mathbf{x}$-space of the polyhedron defined by:

$$
\begin{gathered}
\mathbf{G}^{\{i\}} \mathbf{z}-\mathbf{S}^{\{i\}} \mathbf{x}=\mathbf{W}^{\{i\}}, \quad i \in \mathcal{A}\left(\mathbf{x}^{*}\right), \\
\mathbf{G}^{\{j\}} \mathbf{z}-\mathbf{S}^{\{j\}} \mathbf{x} \leq \mathbf{W}^{\{j\}}, \quad j \in \mathcal{N}\left(\mathbf{x}^{*}\right) .
\end{gathered}
$$

The representation of the critical region $\mathcal{C R}_{k}^{\{l\}}$ (i.e. the projection of (17)) remains the same if the set $\mathcal{N}$ is reduced to those constraints which become active on the facets of the region $\mathcal{C R}_{k}^{\{l\}}$. Consider now only the value function constraints (15c), and, for the moment, assume that only one value function constraint is in the set of active constraints. When computing a critical region $\mathcal{C} \mathcal{R}_{k}^{\{l\}}$, the active value function constraint is known and enforced in (17a). This active value function constraint directly identifies the region $\mathcal{C} \mathcal{R}_{k+1}^{\left\{r^{*}, c\right\}}$ containing the state at time $k+1$. All inactive value function constraints are forced to be inactive by (17b). Geometrically, the value function constraints in $(15 \mathrm{c})$ represent a polyhedra in $(\mathbf{x}, \mathbf{u}, \gamma)$-space, whereby the active value function constraint in (17a) defines one of it's facets. It now follows directly from convexity of the value function $J_{k+1}^{*}$, that all value function constraints which do not originate from regions adjacent to this facet (which corresponds to region $\mathcal{C} \mathcal{R}_{k+1}^{\left\{r^{*}, c\right\}}$ ), are redundant (e.g., see also (14)). Hence, it is sufficient to consider only the value function constraints in $(17 \mathrm{~b})$ which originate from regions adjacent to $\mathcal{C} \mathcal{R}_{k+1}^{\left\{r^{*}, c\right\}}$.

A list of adjacent regions for every critical region can be obtained when solving an mpLP at no additional computational cost (Borrelli, 2003). An example of the solution of an mpLP and the constructed adjacency list is shown on Fig. 2. The approach described above is easily extended

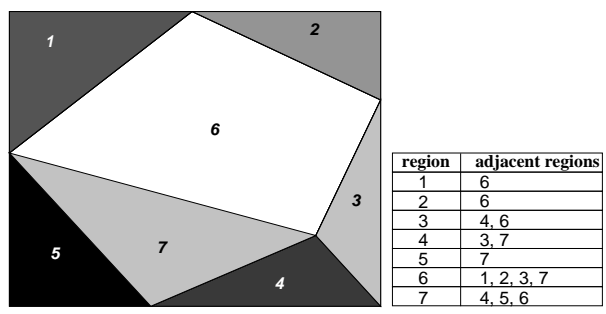

Fig. 2. Critical regions and the corresponding adjacency list

to cases where more than one value function constraint is active. It is then sufficient to consider only those value function constraints in $\mathcal{N}$, which are adjacent to at least one of the active value function constraints.

\subsection{A Note on Complexity}

No tight bounds on the computational complexity of solving multi-parametric programs exist. Hence, it is not possible to perform a detailed complexity comparison of the two algorithms described here. Instead we will discuss the two most crucial aspects of complexity from an intuitive point of view.

Using an affine cost-to-go as in Section 2.2, the CFTOC computation requires the solution to $R_{k+1} \cdot D$ mpLPs, while the proposed algorithm with a piecewise affine cost-to-go solves $C_{k+1} \cdot D$ mpLPs, with an additional variable $\gamma$ and considerably more constraints. If the adjacency scheme in Section 3.2 is applied, the run times for solving the mpLPs do not differ significantly for the two approaches. It always holds that $C_{k+1} \leq R_{k+1}$ and in practice it generally holds that $\bar{C}_{k+1} \ll$ $R_{k+1}$. Hence, fewer mpLPs need to be solved for our algorithm, i.e. the PWA cost-to-go approach.

The other critical component of the CFTOC algorithms in terms of overall runtime is the removal of overlapping partitions, i.e. associating the unique optimal feedback law to each state. On one hand, the affine cost-to-go algorithm solves more mpLPs and is hence likely to produce more controller regions and thus more overlaps. On the other hand, our algorithm solves mpLPs for larger volume target sets, leading to larger volume partitions. 
Thus, it is possible that more controller regions will cover any given state. Therefore, we cannot draw theoretical conclusions on the complexity of overlap removal, although extensive simulations clearly suggest the PWA cost-to-go approach to be superior.

\section{NUMERICAL EXAMPLES}

In order to demonstrate the efficiency of the proposed algorithm, we show the results of an extensive case study. An $\infty$-norm performance objective with $\mathbf{Q}=\mathbf{P}=I$ and $\mathbf{R}=I$ was used in formulating the CFTOC problem. The total computation run times were measured for the proposed algorithm and the algorithm presented in Section 2.2. Both algorithms are implemented using the Multi Parametric Toolbox (Kvasnica et al., 2003) for Matlab. The explicit control laws were computed for 20 randomly generated $2 \mathrm{D}$ PWA systems with 4 affine dynamics and for 20 randomly generated 3D PWA systems with 6 affine dynamics. The horizons $N=7$ and $N=3$ were used for $2 \mathrm{D}$ and $3 \mathrm{D}$ systems respectively. The results are shown on Figure 3 for computations run on a Pentium $4 \mathrm{PC}, 3 \mathrm{GHz}$, using Matlab 6.5 and the NAG LP solver.

\section{CONCLUSION}

In this paper, a novel algorithm was proposed to solve CFTOC problems for discrete-time PWA systems. The algorithm exploits problem structure (i.e. region adjacency information and convexity) to yield faster run times than previously published algorithms. For the analyzed $3^{\text {rd }}$ order PWA systems, the speedup with the new algorithm is typically of one order of magnitude. We cannot claim that the proposed algorithm will outperform alternative schemes in every case, though it was true for all examples studied.

Acknowledgement: We would like to thank Sasikanth Manipatruni for helpful discussions.

\section{REFERENCES}

Baotić, M., F.J. Christophersen and M. Morari (2003). A new Algorithm for Constrained Finite Time Optimal Control of Hybrid Systems with a Linear Performance Index. In: European Control Conference. Cambridge, UK.

Borrelli, F. (2003). Constrained Optimal Control Of Linear And Hybrid Systems. Vol. 290 of Lecture Notes in Control and Information Sciences. Springer.

Dua, V. and E.N. Pistikopoulos (2000). An algorithm for the solution of multiparametric mixed integer linear programming problems. Annals of Operations Research 99, 123-139.

Gal, Tomas (1995). Postoptimal Analyses, Parametric Programming and Related Topics. 2nd ed.. Walter de Gruyter.

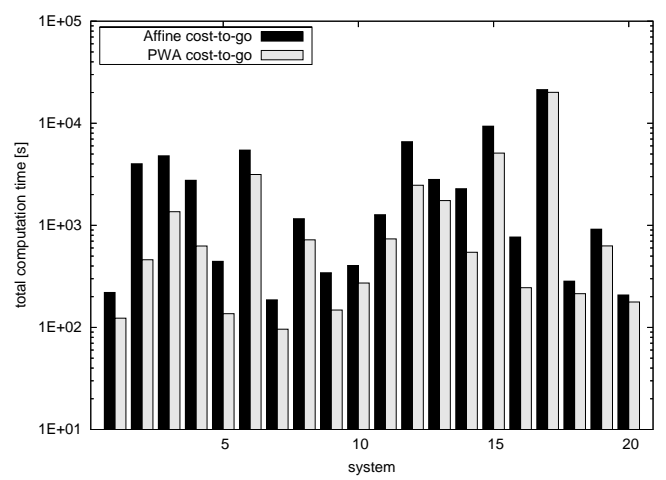

(a) 2D PWA systems with 4 affine dynamics.

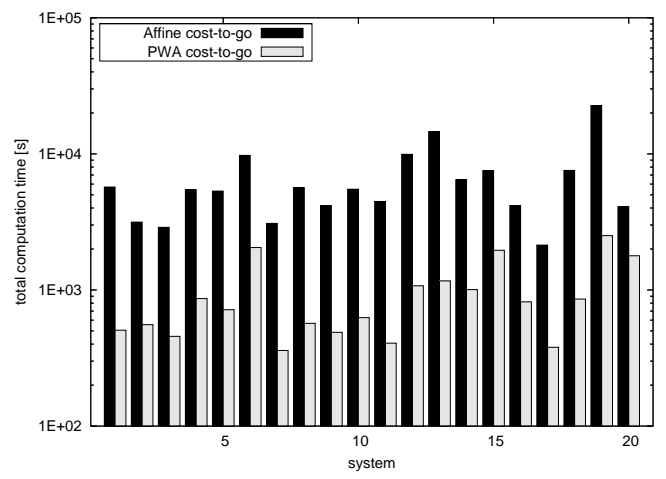

(b) 3D PWA systems with 6 affine dynamics.

Fig. 3. Total computation time for various random PWA systems. Note that the plots are in logscale.

Heemels, W.P.M.H., B. De Schutter and A. Bemporad (2001). Equivalence of hybrid dynamical models. Automatica 37(7), 1085-1091.

Kerrigan, E. C. and D. Q. Mayne (2002). Optimal control of constrained, piecewise affine systems with bounded disturbances. In: Proc. 41st IEEE Conference on Decision and Control. Las Vegas, Nevada, USA.

Kvasnica, M., P. Grieder, M. Baotić and M. Morari (2003). Multi Parametric Toolbox (MPT). In: Hybrid Systems: Computation and Control. Lecture Notes in Computer Science. Springer Verlag. http://control. ee.ethz.ch/ ${ }^{\sim}$ mpt.

Mayne, D. Q. and S. Raković (2002). Optimal control of constrained piecewise affine discrete-time systems using reverse transformation. In: Proc. 41st IEEE Conference on Decision and Control. Las Vegas, Nevada, USA.

Schechter, M. (1987). Polyhedral functions and multiparametric linear programming. Journal of Optimization Theory and Applications 53(2), 269-280.

Sontag, E.D. (1981). Nonlinear regulation: The piecewise linear approach. IEEE Trans. Automatic Control 26(2), 346-358. 